\title{
Comparison of RigiScan and penile color duplex ultrasound in evaluation of erectile dysfunction
}

\author{
Tongyan Liu ${ }^{1}$, Zhihe $\mathrm{Xu}^{2}$, Yong Guan ${ }^{2}$, Mingzhen Yuan ${ }^{2}$ \\ ${ }^{1}$ Department of Ultrasound, the Second Hospital of Shandong University, Jinan, China; ${ }^{2}$ Department of Urology, Shandong Provincial Hospital, \\ Jinan, China \\ Contributions: (I) Conception and design: Y Guan; (II) Administrative support: M Yuan; (III) Provision of study materials or patients: Z Xu; (IV) \\ Collection and assembly of data: Z Xu, T Liu; (V) Data analysis and interpretation: Y Guan; (VI) Manuscript writing: All authors; (VII) Final \\ approval of manuscript: All authors. \\ Correspondence to: Yong Guan. Department of Urology, Shandong Provincial Hospital, Jinan, China. Email: guanyongsdu@163.com.
}

\begin{abstract}
Background: Whether RigiScan could distinguish psychogenic from organic erectile dysfunction (ED) is still a matter of controversy. So this study was aimed to investigate the function of RigiScan in the diagnosis of ED, and compare it with that of pharmacopenile color doppler ultrasonography (PCDU).

Methods: This is a retrospective cohort study. All patients should be performed IIEF-5 questionnaire, blood test, RigiScan, PCDU, Neurophysiological tests. Golden standard: one patient was diagnosed as organic ED when any of the following tests was abnormal, including sex hormone, PCDU and neurological tests. Student t test, Kappa test and ROC analyses was used to analyze the difference between RigiScan and PCDU. Statistical analyses were performed using SPSS 25.0 and EmpowerStats software.

Results: According to the standard: 70 patients (35.4\%) were detected abnormal responses to intracavernous injection in PCDU and normal responses in 128 cases (64.6\%). RigiScan had a significant but weak positive correlation with PCDU test (kappa value $=0.361, \mathrm{P}<0.01$ ). In the ROC analyses, RigiScan was better in sensitivity (0.8603 vs. 0.5147$)$. In the patients revealed organic lesions by RigiScan, 61 of $119(51.3 \%)$ patients were detected abnormal responses by PCDU.

Conclusions: RigiScan was the preferential choice in distinguishing psychogenic ED from organic ED. PCDU could not replace RigiScan by now. But PCDU was the preferential choice in ED with vascular lesions.
\end{abstract}

Keywords: Erectile dysfunction (ED); RigiScan; doppler ultrasonography

Submitted Feb 29, 2020. Accepted for publication Aug 02, 2020.

doi: 10.21037/apm-20-507

View this article at: http://dx.doi.org/10.21037/apm-20-507

\section{Introduction}

Erectile dysfunction (ED) was defined as the inability to achieve or maintain an erection adequate for sexual satisfaction (1). And the prevalence rate of ED is estimated to be $10 \%$ to $20 \%$ worldwide $(2,3)$. After the introduction of new oral drugs for the treatment of ED, most clinicians adopted a goal-oriented approach to treat most ED patients (4). However, the diagnostic approach should be individualized as impotence is not a life threatening but a functional disease. So revealing the underlying etiology of $\mathrm{ED}$ is very important for individual treatment.
ED could be divided into psychologic and organic ED by etiology, in which organic ED may be caused by vasculogenic, neurogenic or other factors. Nocturnal penile tumescence (NPT) monitoring was the acknowledged objective test for diagnosis of ED, which could distinguish psychogenic from organic impotence (5). Its' function in male impotence was first reported by Fisher et al., who described that penile erections naturally occur 3-5 times during sleep (6). The RigiScan device (Dacomed Corporation, Minneapolis, MN, USA) is the most reliable test to be used in NPT test (7-9). 
However, whether RigiScan could distinguish psychogenic from organic ED is still a matter of controversy $(10,11)$. And the absence of nocturnal erections does not indicate the etiology of the ED, even if due to an organic cause (12). Some researchers thought that pharmacopenile color doppler ultrasonography (PCDU) may replace the NPT test because PCDU was repeatable and most organic $\mathrm{ED}$ was vascular origin.

So in this study, we investigated the function of RigiScan in the diagnosis of $\mathrm{ED}$, and we also compare it with that of PCDU.

We present the following article in accordance with the STROBE reporting checklist (available at http://dx.doi. org/10.21037/apm-20-507).

\section{Methods}

A retrospective cohort study was performed. One hundred and ninety eight patients in the second hospital of Shandong university between January 2009 and October 2015 because of ED were enrolled in our research. Every patient signed consent after taking the approval of our local ethical committee, Men were included in our study if their ED history is greater than 6-month duration and patients were excluded if they have one of the following diseases: spinal cord injuries, concurrent neurologic disease, morbid obesity, some drugs to affect erectile function, severe heart disease, penile fibrosis, hypertension. Patients who were included in this study underwent the following tests.

All procedures performed in studies involving human participants were in accordance with the ethical standards of the Second Hospital of Shandong University (No. 2019-156) and with the 1964 Helsinki declaration (as revised in 2013) and its later amendments or comparable ethical standards. Informed consent was obtained from all individual participants included in the study.

\section{NPT test}

NPT tests were performed using the RigiScan ${ }^{\circledR}$ device in all the patients. To ensure good night sleep and less affection the patients were asked to avoid caffeine or alcohol intake and evacuate the bladder before going to sleep. Data was collected in the next morning. The test was conducted three consecutive nights for avoiding the "first night effect". The test results were considered normal if at least one nocturnal erection event fulfills the following criteria: tip and base rigidity $\geq 70 \%$, an increase in tumescence at the tip $\geq 2 \mathrm{~cm}$ and at the base $\geq 3 \mathrm{~cm}$, and if the event lasts $\geq 10$-minute duration $(9,13,14)$.

\section{Duplex ultrasonography}

This test was performed with a GE color duplex ultrasound machine (GE LOGIQ9, America). Patients received a single intracavernous injection of Trimix (15 $\mathrm{mg}$ papaverine $5 \mathrm{mg}$ prostaglandin E1, and $1 \mathrm{mg}$ phentolamine). The erection was evaluated by palpation of the penis and the rigidity was divided into four levels. The penis was scanned from the ventral part at the base. Peak systolic velocity (PSV) and end diastolic velocity (EDV) within the cavernosal arteries were detected. Patients with PSV more than $35 \mathrm{~cm} / \mathrm{s}$ were considered normal arterial response, while less than $25 \mathrm{~cm} / \mathrm{s}$ demonstrated arterial dysfunction. Corporal venoocclusive dysfunction was defined as EDV $>5 \mathrm{~cm} / \mathrm{s}$ and RI $<0.85(15-18)$.

\section{Neurophysiological tests}

Posterior tibial somatosensory nerve evoked potentials (PTSSEPs), pudendal nerve evoked potentials (PDEPs) and the bulbocavernosus reflex (BCR) test were performed for all the patients. PTSSEPs and PDEPs were conducted according to the International Federation of Clinical Neurophysiology (IFCN) standards (19). The latency of cortical $\mathrm{P} 40>45 \mathrm{~ms}$ or left-right difference $>2.5 \mathrm{~ms}$ was considered abnormal response in the PTSSEPs test. PDEPs test was considered abnormal if P40 latency $>44.1 \mathrm{~ms}$. BCR test was conducted by applying electrical pulses and the responses were recorded from both bulbocavernosus muscles with concentric needle electrodes $(20,21)$. Abnormal results were defined as absent responses, response latency $>37 \mathrm{~ms}$ and interside differences $>1.5 \mathrm{~ms}$ (15).

\section{Golden standard}

There is no perfect golden standard for organic ED by now. In this study, one patient was diagnosed as organic ED when any one of the following tests was abnormal, including sex hormone, PCDU and neurological tests.

\section{Statistical analyses}

Student $t$ test was used to test the differences between the groups. Kappa test was done to determine the agreement between these two methods. ROC analyses were used 
Table 1 Clinical characteristics of these patients for standard 1

\begin{tabular}{lcc}
\hline Variable & Psychologic ED & Organic ED \\
\hline $\mathrm{N}$ & 62 & 136 \\
Age (years old) & $27.5 \pm 4.7$ & $30.3 \pm 8.3$ \\
Glucose (mmol/L) & $4.7 \pm 0.5$ & $5.1 \pm 1.1$ \\
Triglyceride & $1.3 \pm 1.1$ & $1.2 \pm 0.7$ \\
History of smoking & & \\
+ & $12(19.4 \%)$ & $23(16.9 \%)$ \\
- & $50(80.6 \%)$ & $113(83.1 \%)$ \\
History of diabetes & & $4(2.9 \%)$ \\
+ & 0 & $132(97.1 \%)$ \\
- & $62(100.0 \%)$ & \\
Sex hormone & & $47(69.6 \%)$ \\
+ & $39(62.9 \%)$ & \\
\hline
\end{tabular}

Basic characteristic of patients. Data was showed as mean \pm SD. ED, erectile dysfunction.

Table 2 results of RigiScan and PCDU test: standard 1

\begin{tabular}{|c|c|c|c|c|c|c|}
\hline \multirow{3}{*}{ RigiScan } & \multicolumn{3}{|c|}{ Psychogenic ED } & \multicolumn{3}{|c|}{ Organic ED } \\
\hline & \multicolumn{3}{|c|}{ PCDU } & \multicolumn{3}{|c|}{ PCDU } \\
\hline & + & - & Sum & + & - & Sum \\
\hline+ & 0 & 2 & 2 & 61 & 56 & 117 \\
\hline- & 0 & 60 & 60 & 9 & 10 & 19 \\
\hline Sum & 0 & 62 & 62 & 70 & 66 & 136 \\
\hline
\end{tabular}

to compare the efficacy of these two methods. Data was considered statistically significant at $\mathrm{P}<0.05$. Values reported are mean \pm standard deviation (SD). Statistical analyses were performed using SPSS 25.0 and EmpowerStats software.

\section{Results}

\section{Clinical characteristics}

The clinical characteristics of patients for standard were shown as Table 1. Some basic characteristics, such as age, glucose, triglyceride, history of smoking and diabetes, sex
Table 3 Correlation test for RigiScan and PCDU in organic ED

\begin{tabular}{lcc}
\hline & PCDU (+) & PCDU (-) \\
\hline RigiScan (+) & 61 & 58 \\
RigiScan (-) & 9 & 70
\end{tabular}

Kappa value $=0.361, \mathrm{P}<0.01$. PCDU, pharmacopenile color doppler ultrasonography; ED, erectile dysfunction.

Table 4 ROC analyses for RigiScan and PCDU

\begin{tabular}{lcc}
\hline & Sensitivity & Speciality \\
\hline RigiScan & 0.8603 & 0.9677 \\
PCDU & 0.5147 & 1.0000 \\
\hline PCDU, pharmacopenile color doppler ultrasonography.
\end{tabular}

hormone, were compared. There was no great statistical significance.

\section{RigiScan and PCDU test}

Cases found to be of psychogenic origin were 62 (31.31\%) and of organic origin were $136(68.69 \%)$ by all the examination. In the patients with psychogenic ED, 60 (96.77\%) patients had normal nocturnal penile erection by RigiScan test. In the patients with organic ED. PCDU detected abnormal responses to intracavernous injection of Timix in 70 patients $(51.5 \%)$ and normal responses in 66 cases $(48.5 \%)$ (Table 2).

Meanwhile, 117 (86.0\%) patients had abnormal performance in RigiScan test. $\chi^{2}=31.299, \mathrm{P}=0.001$. The results have great statistical significance. And RigiScan had a significant but weak positive correlation with PCDU test (kappa value $=0.361, \mathrm{P}<0.01$, Table 3).

To detect which is most predictive of the psychogenic $\mathrm{ED} v s$. organic ED, the ROC analysis was done. In the ROC analyses, RigiScan was better in sensitivity (0.8603 vs. 0.5147 ) (Table 4).

\section{Discussion}

RigiScan is traditionally used to differentiate between psychogenic and organic ED (4). As the RigiScan is a noninvasive technique, some researchers tried to use it in differentiating subtypes of organic ED especially the vasculogenic types and they correlated its results with other diagnostic modalities $(22,23)$. 
However, whether RigiScan could distinguish psychogenic from organic ED is still a matter of controversy (24). And some urologists thought it could be substituted by PCDU. The main controversy focused on following points. First, for RigiScan, no reports could prove that RigiScan could distinguish psychogenic from organic ED and it could not find the cause the ED (12). Patients should be in hospital, so lots of factors may impact the results and has poor repeatability, patients should pay high medical fee. Second, for PCDU, most ED patients was diagnosed as vasculogenic ED and PCDU could identify the vascular abnormality (25). Few factors could affect the results and it has good repeatability.

So in this study, we compared the diagnostic efficiency between RigiScan and PCDU and we found few similar studies. We found that both RigiScan and PCDU have its advantages and neither should be replaced.

"Golden standard" should be established if we performed the study to compare these two methods. For this study, this is the first choice for golden standard because these methods could find the organic problems directly. The true negative rate of RigiScan was $96.77 \%$. The true of positive rate was $86.03 \%$ of RigiScan vs. $51.47 \%$ of PCDU. RigiScan had a significant but weak positive correlation with PCDU test (kappa value $=0.361, \mathrm{P}<0.01$ ). So from this standard, we could demonstrate that RigiScan has its advantage compared with PCDU, but there are still more than $10 \%$ patients who couldn't be diagnosed rightly.

The possible explanation about the results that these two tests were not matched was the following three aspects. First, the criteria of RigiScan were not accorded with that of PCDU. So the criteria were achieved in RigiScan in the night, but the PSV or EDV was not up to the standard of PCDU. Second, there is some limitation of RigiScan in the diagnosis of organic ED and psychogenic ED. So there may be some errors in the process of diagnosis. Thirdly, the vascular reactivity was normal, but the drugs performed direct action to vasculature and no neurotransmitter was released. So this demonstrated that there may be some problems with neural conduction or neurotransmitters.

So we could conclude that RigiScan has more advantages in distinguishing psychogenic ED from organic ED than that of PCDU, but it still should be completed with other methods.

\section{Conclusions}

RigiScan was the preferential choice in distinguishing psychogenic ED from organic ED. It has more advantages over PCDU. PCDU could not replace RigiScan by now. The diagnosis organic ED still should be performed multiple examinations for synthetical judgments.

\section{Acknowledgments}

Funding: This work was supported by the National Nature Science Foundation of China (81900613), Shandong Province Natural Science Foundation (ZR2018BH007, 2019GSF108116), the youth found and youth talent found of the second hospital of Shandong University (Y2015010038).

\section{Footnote}

Reporting Checklist: The authors have completed the STROBE reporting checklist. Available at http://dx.doi. org/10.21037/apm-20-507

Data Sharing Statement: Available at http://dx.doi. org/10.21037/apm-20-507

Conflicts of Interest: All authors have completed the ICMJE uniform disclosure form (available at http://dx.doi. org/10.21037/apm-20-507). The authors have no conflicts of interest to declare.

Ethical Statement: The authors are accountable for all aspects of the work in ensuring that questions related to the accuracy or integrity of any part of the work are appropriately investigated and resolved. All procedures performed in studies involving human participants were in accordance with the ethical standards of the Second Hospital of Shandong University (No. 2019-156) and with the 1964 Helsinki declaration (as revised in 2013) and its later amendments or comparable ethical standards. Informed consent was obtained from all individual participants included in the study.

Open Access Statement: This is an Open Access article distributed in accordance with the Creative Commons Attribution-NonCommercial-NoDerivs 4.0 International License (CC BY-NC-ND 4.0), which permits the noncommercial replication and distribution of the article with the strict proviso that no changes or edits are made and the original work is properly cited (including links to both the formal publication through the relevant DOI and the license). See: https://creativecommons.org/licenses/by-nc-nd/4.0/. 


\section{References}

1. Morgentaler A. Male impotence. Lancet 1999;354:1713-8.

2. Derogatis LR, Burnett AL. The epidemiology of sexual dysfunctions. J Sex Med 2008;5:289-300.

3. Johannes CB, Araujo AB, Feldman HA, et al. Incidence of erectile dysfunction in men 40 to 69 years old: longitudinal results from the Massachusetts male aging study. J Urol 2000;163:460-3.

4. Elhanbly S, Elkholy A, Elbayomy Y, et al. Nocturnal penile erections: the diagnostic value of tumescence and rigidity activity units. Int J Impot Res 2009;21:376-81.

5. Marshall P, Morales A, Surridge D. Diagnostic significance of penile erections during sleep. Urology 1982;20:1-6.

6. Fisher C, Gorss J, Zuch J. Cycle of Penile Erection Synchronous with Dreaming (Rem) Sleep. Preliminary Report. Arch Gen Psychiatry 1965;12:29-45.

7. Bradley WE, Timm GW, Gallagher JM, et al. New method for continuous measurement of nocturnal penile tumescence and rigidity. Urology 1985;26:4-9.

8. Hatzichristou DG, Hatzimouratidis K, Ioannides E, et al. Nocturnal penile tumescence and rigidity monitoring in young potent volunteers: reproducibility, evaluation criteria and the effect of sexual intercourse. J Urol 1998;159:1921-6.

9. Levine LA, Lenting EL. Use of nocturnal penile tumescence and rigidity in the evaluation of male erectile dysfunction. Urol Clin North Am 1995;22:775-88.

10. Ghanem H, Shamloul R. An evidence-based perspective to commonly performed erectile dysfunction investigations. J Sex Med 2008;5:1582-9.

11. Meisler AW, Carey MP. A critical reevaluation of nocturnal penile tumescence monitoring in the diagnosis of erectile dysfunction. J Nerv Ment Dis 1990;178:78-89.

12. Sohn MH, Seeger U, Sikora R, et al. Criteria for examinerindependent nocturnal penile tumescence and rigidity monitoring (NPTR): correlations to invasive diagnostic methods. Int J Impot Res 1993;5:59-68.

13. Cilurzo P, Canale D, Turchi P, et al. The Rigiscan system in the diagnosis of male sexual impotence. Arch Ital Urol Nefrol Androl 1992;64 Suppl 2:81-5.

14. Morales A, Condra M, Reid K. The role of nocturnal penile tumescence monitoring in the diagnosis of impotence: a review. J Urol 1990;143:441-6.

15. Guan Y, Wendong S, Zhao S, et al. The vascular and neurogenic factors associated with erectile dysfunction in patients after pelvic fractures. Int Braz J Urol
2015;41:959-66.

16. Kim SC. Recent advancement in diagnosis of vasculogenic impotence. Asian J Androl 1999;1:37-43.

17. Shenfeld OZ, Kiselgorf D, Gofrit ON, et al. The incidence and causes of erectile dysfunction after pelvic fractures associated with posterior urethral disruption. J Urol 2003;169:2173-6.

18. Sikka SC, Hellstrom WJ, Brock G, et al. Standardization of vascular assessment of erectile dysfunction: standard operating procedures for duplex ultrasound. J Sex Med 2013;10:120-9.

19. Nuwer MR, Aminoff M, Desmedt J, et al. IFCN recommended standards for short latency somatosensory evoked potentials. Report of an IFCN committee. International Federation of Clinical Neurophysiology. Electroencephalogr Clin Neurophysiol 1994;91:6-11.

20. Ertekin C, Reel F. Bulbocavernosus reflex in normal men and in patients with neurogenic bladder and/or impotence. J Neurol Sci 1976;28:1-15.

21. Valles-Antuña C, Fernandez-Gomez J, FernandezGonzalez F. Peripheral neuropathy: an underdiagnosed cause of erectile dysfunction. BJU Int 2011;108:1855-9.

22. Basar MM, Atan A, Tekdogan UY. New concept parameters of RigiScan in differentiation of vascular erectile dysfunction: is it a useful test? Int J Urol 2001;8:686-91.

23. Wylie KR, Davies-South D, Steward D, et al. A comparison between portable ultrasound (MIDUS) and nocturnal RigiScan when confirming the diagnosis of vascular organic erectile disorder. Int J Impot Res 2006;18:354-8.

24. Jannini EA, Granata AM, Hatzimouratidis K, et al. Use and abuse of Rigiscan in the diagnosis of erectile dysfunction. J Sex Med 2009;6:1820-9.

25. Jefferson TW, Glaros A, Spevack M, et al. An evaluation of the Minnesota Multiphasic Personality Inventory as a discriminator of primary organic and primary psychogenic impotence in diabetic males. Arch Sex Behav 1989;18:117-26.

Cite this article as: Liu T, Xu Z, Guan Y, Yuan M. Comparison of RigiScan and penile color duplex ultrasound in evaluation of erectile dysfunction. Ann Palliat Med 2020;9(5):29882992. doi: 10.21037/apm-20-507 